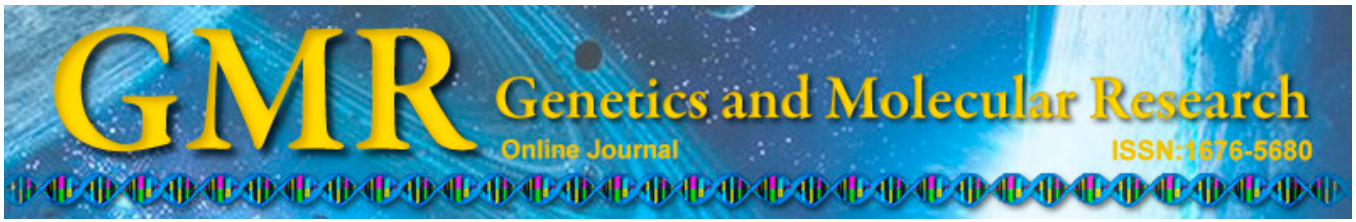

\title{
Role of adiponectin in adipose tissue wound healing
}

\author{
C.E. Jin ${ }^{1 *}$, L. Xiao ${ }^{2 *}$, Z.H. Ge ${ }^{2}$, X.B. Zhan ${ }^{2}$ and H.X. Zhou ${ }^{2}$ \\ ${ }^{1}$ Department of Respiratory Medicine, Shenzhen People's Hospital, \\ Second Clinical Medical College of Jinan University, Shenzhen, \\ Guangdong, China \\ ${ }^{2}$ Department of Surgery and Biological Therapy, \\ Shenzhen Second People's Hospital, First Affiliated Hospital of Shenzhen University, \\ Shenzhen, Guangdong, China \\ *These authors contributed equally to this study. \\ Corresponding authors: L. Xiao / C.E. Jin \\ E-mail: xiaoliang_b@126.com / 9179320@qq.com
}

Genet. Mol. Res. 14 (3): 8883-8891 (2015)

Received December 9, 2014

Accepted April 29, 2015

Published August 3, 2015

DOI http://dx.doi.org/10.4238/2015.August.3.11

\begin{abstract}
The purpose of this study was to investigate the mechanism behind adipose tissue wound healing (ATWH). The preadipocyte cell line 3T3-L1 was cultured and expression of adiponectin receptors (AdipoR1/2) was detected by immunohistochemistry and reverse transcription polymerase chain reaction. The concentration of adiponectin secreted at different cell densities was measured by enzyme-linked immunosorbent assay, while preadipocyte proliferation and migration were determined in vitro by MTT and wound closure assays. AdipoR1/2 were found to be expressed in 3T3-L1 preadipocytes. There were no statistically significant differences in the concentrations of adiponectin secreted by cell solutions of different densities $(\mathrm{P}>0.05)$. In addition, adiponectin was seen to promote the growth and migration of preadipocytes. In conclusion, adiponectin may regulate ATWH by promoting preadipocyte proliferation and migration, and its systemic and/or local application is proposed as a
\end{abstract}


promising therapeutic approach for the treatment of wounds incurred as a result of surgery.

Key words: Wound healing; Adiponectin; Adipose tissue; Preadipocyte; Proliferation; Migration

\section{INTRODUCTION}

The poor healing of incisions made during surgery constitutes the most common abdominal postoperative complication, with an incidence rate of 7 to $26 \%$ (Jeon et al., 2011). Fat necrosis is also common and mainly occurs in overweight and obese patients. The typical wound healing process includes inflammatory, proliferative, and remodeling phases (Pradhan et al., 2011). Studies of wound models have predominately concentrated on impaired wound healing in diabetes, with a specific focus on scar formation, epithelial stem cell transformation, angiogenesis, and inflammation control (Lu et al., 2009; Liang et al., 2009; Cheng et al., 2011). Little research into adipose tissue wound healing (ATWH) has been carried out.

Adiponectin is an endogenous bioactive protein secreted by adipose cells (Kawada and Hasegawa, 2012) that performs a variety of roles in different tissue and cell types. For example, it has been implicated in insulin sensitivity, the anti-inflammatory response, and in mitigating the effects of atherosclerosis (Maeda et al., 2002; Okamoto et al., 2002, 2006). Besides, mice lacking adiponectin show impaired wound healing and reduced keratinocyte proliferation and migration. Cutaneous healing in adiponectin-deficient mice improved following treatment with adiponectin, while no obvious effect of such treatment was observed in normal controls, revealing that adiponectin promotes wound healing (Shibata et al., 2012). However, adiponectin's function in ATWH remains unclear.

The aim of this study was to investigate the role of adiponectin in ATWH. We first established cultures of the mouse preadipocyte line 3T3-L1, as a model of ATWH, and subsequently tested the effect of adiponectin on the proliferation and migration of these cells. We aimed to provide an experimental basis for the use of adiponectin in the treatment of postoperative complications caused by cutaneous wounds.

\section{MATERIAL AND METHODS}

\section{In vitro culture of the mouse preadipocyte line 3T3-L1}

Mouse preadipocytes were purchased from the American Type Culture Collection (Manassas, VA, USA) and were routinely maintained in a growth medium of Dulbecco's modified Eagle's medium (DMEM, St. Louis, MI, USA) supplemented with $10 \%$ fetal bovine serum (FBS, Gibco, Thermo Fisher Scientific Inc.) at $37^{\circ} \mathrm{C}$ in a humidified atmosphere of $5 \% \mathrm{CO} 2$. The medium was changed every 2 days and cell monolayers were detached with $0.25 \%$ trypsin (Gibco, Thermo Fisher Scientific Inc.) prior to being passaged. Passaging was carried out at regular intervals until cells were used in experiments. Preadipocyte morphology was observed using an inverted microscope (IX83, Olympus, Japan) and images were recorded. 


\section{Inducing differentiation of mouse preadipocytes}

All reagents were purchased from Sigma (St. Louis, MI, USA) unless stated otherwise. Differentiation of 3T3-L1 preadipocytes was induced using the typical hormone cocktail method. Cells $\left(6 \times 10^{4}\right.$ cells/well) were plated on 6-well plates, and a sterilized cover glass was placed on each well. Then, cells were cultured in DMEM containing $10 \%$ FBS in a humidified $5 \% \mathrm{CO}_{2}$ atmosphere at $37^{\circ} \mathrm{C}$. Two days after the cells had reached confluence, differentiation was induced by replacing the growth medium with an induction medium comprising DMEM, $0.5 \mathrm{mM} 3$-isobutyl-1-methylxanthine (IBMX), $10 \mu \mathrm{g} / \mathrm{mL}$ insulin (INS), $1 \mu \mathrm{M}$ dexamethasone (DEX), and 10\% FBS. After 2 days, the induction medium was replaced by DMEM containing $10 \mu \mathrm{g} / \mathrm{mL}$ INS and $10 \%$ FBS. After a further 2 days, this was substituted with fresh growth medium, which was replaced at 2-day intervals until day 8 , at which point $90 \%$ cells appeared to have a phenotype typical of adipocytes.

\section{Oil red O staining}

On day 8 , slides of cells were washed 3 times with phosphate buffered saline (PBS), and fixed with $4 \%$ formaldehyde-phosphate buffer for $1 \mathrm{~h}$, before being rinsed 3 times with water and stained for $1 \mathrm{~h}$ with $60 \%$ isopropanol containing $0.5 \%$ oil red O (Beijing Solarbio Science \& Technology Co., Ltd., Beijing, China). Cells were then counterstained with hematoxylin and washed with PBS. Images of cells were subsequently taken under a microscope.

\section{Immunohistochemistry analysis of adiponectin receptor expression (AdipoR1/2)}

Firstly, cell slides were made by placing a suspension containing $1 \times 10^{6}$ cells $/ \mathrm{mL}$ on sterilized cover glasses and leaving them for $30 \mathrm{~min}$. Then, growth medium was added and cells were incubated in a humidified $5 \% \mathrm{CO}_{2}$ atmosphere at $37^{\circ} \mathrm{C}$ for 3 to $6 \mathrm{~h}$. Cells were observed under a microscope to verify that they had attached to the slides, prior to being fixed in $4 \%$ formaldehyde-phosphate buffer for $30 \mathrm{~min}$. This was followed by a $30 \mathrm{~min}$, room temperature incubation in $30 \% \mathrm{H}_{2} \mathrm{O}_{2}$ diluted 1 in 50 in pure methanol, and 3 washes with PBS, each lasting for 2 to $3 \mathrm{~min}$. The slides were then blocked with 5\% bovine serum albumin for $20 \mathrm{~min}$. Monoclonal rabbit anti-AdipoR1 or polyclonal goat anti-AdipoR2 primary antibodies, diluted 1 in 200 , were incubated with cells overnight at $4^{\circ} \mathrm{C}$. Goat anti-rabbit and donkey anti-goat secondary antibodies were subsequently added and slides were kept at $37^{\circ} \mathrm{C}$ for $20 \mathrm{~min}$. Cells were then incubated with streptavidin-biotin complex at $37^{\circ} \mathrm{C}$ for a further $20 \mathrm{~min}$. Finally, slides were stained with 3,3'-diaminobenzidine and counterstained with hematoxylin before being mounted and observed under a microscope. For the control group, PBS was used in place of primary antibodies. All antibodies were purchased from Abcam (Cambridge, MA, USA).

\section{Reverse transcription PCR (RT-PCR) analysis of AdipoR1/2 expression}

Total RNA was isolated using an RNAsimple Total RNA kit (TIANGEN Biotech Co., Ltd., Beijing, China) and cDNA synthesized with a ReverTra Ace qPCR RT Kit (Toyobo, Osaka, Japan). Conventional PCR reactions were then carried out with the following primers specific for AdipoR1: forward, 5'-GACAGGCCTAAGTGTCCAT-3'; and reverse, 5'-GCATGCTTGTTCTTCT-3'. The primers used to amplify AdipoR2 were as follows: forward, 
5'-TGACAACCACCACGGAGA-3'; and reverse, 5'-GTCCAAATGTTGCCCGTC-3'. All primers were synthesized by BGI Tech (BGI Tech Solutions Co., Ltd. Shenzhen, China). Thermal cycling was carried out with a 5 min denaturation step at $94^{\circ} \mathrm{C}$, followed by 40 cycles of $30 \mathrm{~s}$ at $94^{\circ} \mathrm{C}, 30 \mathrm{~s}$ at $60^{\circ} \mathrm{C}$, and $60 \mathrm{~s}$ at $68^{\circ} \mathrm{C}$. PCR products were separated by electrophoresis on a $1 \%$ agarose gel and visualized under ultraviolet light.

\section{Enzyme-linked immunosorbent assay (ELISA) analysis of adiponectin secreted by different densities of 3T3-L1 cells}

Preadipocyte cells were seeded on 6-well plates at a density of $5 \times 10^{5}$ cells/well and maintained in a humidified $5 \% \mathrm{CO}_{2}$ atmosphere at $37^{\circ} \mathrm{C}$ for $12,24,48$, and $72 \mathrm{~h}$, over which periods it was not necessary to replace the culture medium. Cells were counted after each period and the concentration of adiponectin in the culture supernatant was quantified using an ELISA kit (Boster, Wuhan, China), according to the manufacturer protocol. Enzyme-labelling measuring instrument (SP-Max 3500FL, Shanghai Flash Spectrum Biological Technology Co., LTD) was used to measure the absorbance value of $450 \mathrm{~nm}$.

\section{Influence of adiponectin on cell proliferative activity measured by 3-(4,5-dimethylthiazol-2-yl)-2,5-diphenyltetrazolium bromide (MTT) assay}

Cells were seeded on 96-well plates at a density of $6 \times 10^{4}$ cells/well and cultured in a humidified $5 \% \mathrm{CO}_{2}$ atmosphere at $37^{\circ} \mathrm{C}$ for $3 \mathrm{~h}$. Then, cells were divided into 6 groups, and each group into 3 wells, and the following concentrations of adiponectin (CYT-280; ProSpec, East Brunswick, NJ, USA) were added: 0.625, 1.25, 2.5, 5, and $10 \mathrm{ng} / \mathrm{mL}$. For the control group, culture medium was added in place of adiponectin. Cells were then cultured in a humidified $5 \% \mathrm{CO}_{2}$ atmosphere at $37^{\circ} \mathrm{C}$ for $72 \mathrm{~h}$. Following this, $20 \mu \mathrm{L} 5 \mathrm{mg} / \mathrm{mL}$ MTT was added to each well and after $4 \mathrm{~h}$, cell proliferation was estimated according to the manufacturer protocol, with absorbance being measured at $490 \mathrm{~nm}$.

\section{Influence of adiponectin on cell migration measured by wound healing}

Preadipocyte cells were seeded on 6-well plates at a density of $5 \times 10^{5}$ cells/well and cultured in a humidified $5 \% \mathrm{CO}_{2}$ atmosphere at $37^{\circ} \mathrm{C}$ for $24 \mathrm{~h}$, during the time confluency of cell monolayers was 70 to $80 \%$. The single layer of cells was then lightly scratched with a 10 $\mu \mathrm{L}$ volume aseptic pipette tip and plates were washed twice with PBS to remove loose cells. New culture medium was added, and cultures were divided randomly into 3 groups: 10\% DMEM; $10 \%$ DMEM + 10\% FBS; and 10\% DMEM $+10 \%$ FBS $+25 \mu \mathrm{g} / \mathrm{mL}$ adiponectin. Wound healing status was then monitored at $0,6,12$, and $24 \mathrm{~h}$ after scratching.

\section{Statistical analysis}

Statistical analysis was performed using SPSS version 17.0 (SPSS Inc., Chicago, IL, USA). Data are reported as means \pm standard deviation (SD). The $t$-test was used to compare 2 groups, while one-way ANOVA analysis was used for comparisons between more than 2 groups. Differences with P values lower than or equal to 0.05 were considered to be statistically significant. 


\section{RESULTS}

\section{Culture and maturation of 3T3-L1 preadipocytes}

Preadipocyte morphology was observed under an inverted microscope after 3 passages. Cells were of uniform shape and size, with a spindle-like form and large projections. Their appearance was homogeneous and fibroblast-like, and they were seen to adhere to the wall of the container (Figure 1A). Preadipocytes began to transform into adipocytes after treatment with IBMX + INS + DEX. Cell volumes increased gradually and their shape changed from spindle-like to elliptic, quasi-circular, and round. After 8 days of differentiation, the 3T3-L1 preadipocytes were dyed with oil red $\mathrm{O}$, which revealed the merging of smaller lipid drops into larger ones. By this stage, most cells demonstrated adipocyte morphology, and our results suggest that cells had indeed differentiated (Figure 1B).
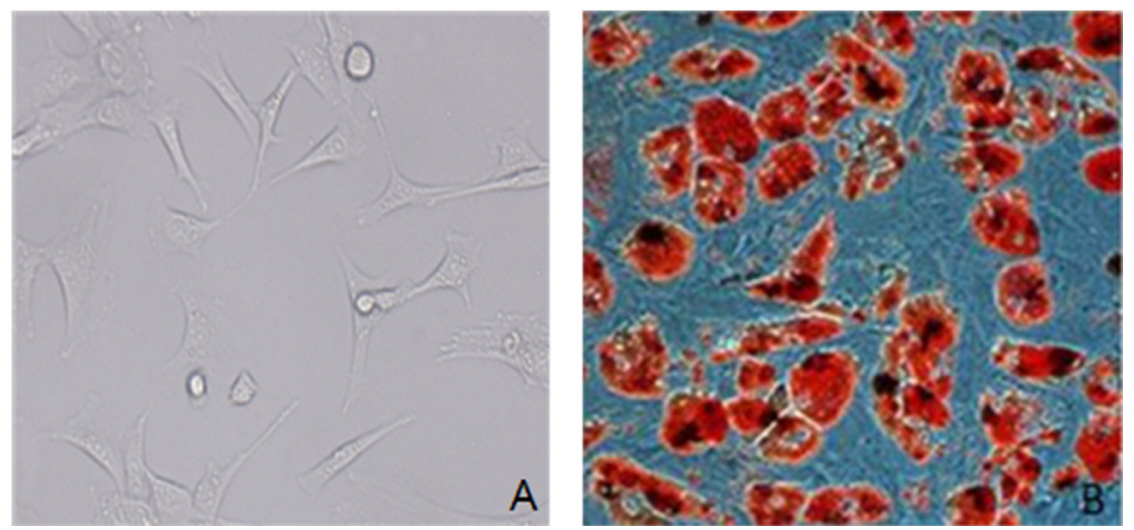

Figure 1. Morphology of 3T3-L1 preadipocytes before and after differentiation. (A) Undifferentiated 3T3-L1 preadipocytes; (B) mature adipocytes identified by oil red $\mathrm{O}$ staining.

\section{Expression of AdipoR1/2 in 3T3-L1 preadipocytes}

Immunohistochemistry revealed the expression of AdipoR1/2 in 3T3-L1 preadipocytes, which was consistent with our RT-PCR results (Figure 2).
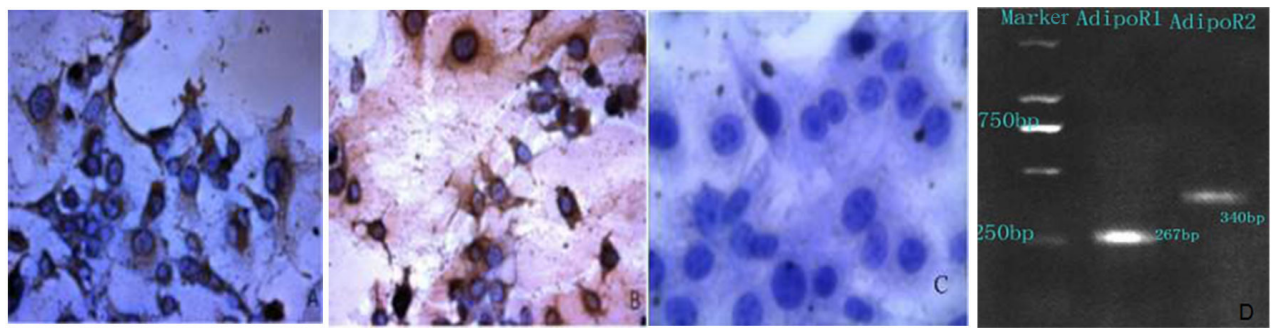

Figure 2. Expression of AdipoR1/2 in 3T3-L1 preadipocytes identified by immunohistochemistry. (A) AdipoR1 positive cells, 3,3'-diaminobenzidine (DAB) staining, 100X magnification; (B) AdipoR2 positive cells, DAB staining, 100X magnification; (C) PBS-treated control group, DAB staining, 200X magnification; (D) AdipoR1 and AdipoR2 polymerase chain reaction products were in accordance with immunohistochemistry results. 


\section{Adiponectin concentration in various densities of 3T3-L1 preadipocytes}

Cell counts of 3T3-L1 preadipocytes cultured on 6-well plates $(2 \mathrm{~mL})$ for $12,24,48$, and $72 \mathrm{~h}$ were $6 \times 10^{5}, 9.9 \times 10^{5}, 12.3 \times 10^{5}$, and $13.3 \times 10^{5}$ respectively. Optical density values as a measure of adiponectin concentration were $0.05515 \pm 0.00025,0.05835 \pm 0.00021$, $0.05625 \pm 0.00092$, and $0.05855 \pm 0.00049$ respectively, while that for the control group, in which cell density was 0 , was $0.05175 \pm 0.00304$. Differences between each group were not statistically significant, ensuring the legitimacy of subsequent experimental results.

\section{Influence of adiponectin on 3T3-L1 preadipocyte cell proliferation}

Treatment with adiponectin increased the number of active preadipocytes in a dosedependent manner at lower doses. However, at concentrations above $1.25 \mathrm{ng} / \mathrm{mL}$, this promotive effect was reduced (Figure 3).

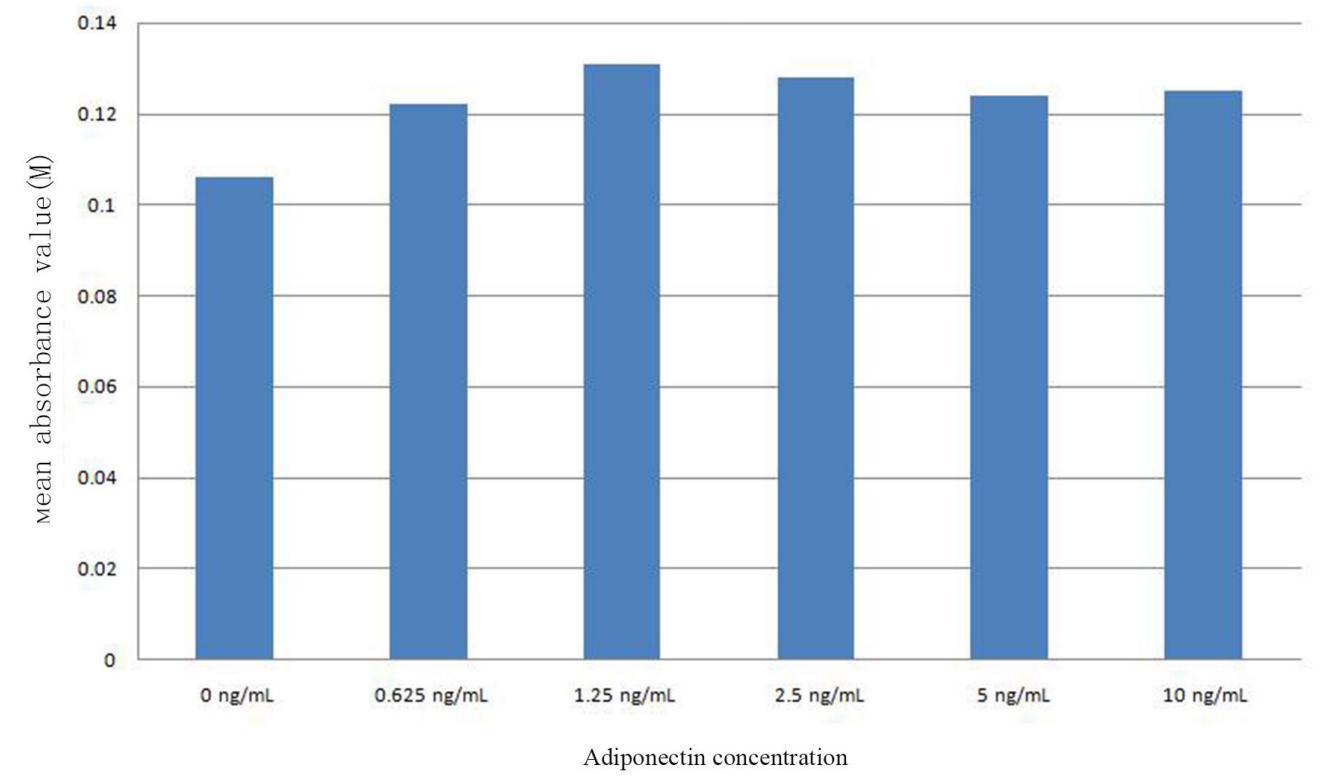

Figure 3. Influence of adiponectin on 3T3-L1 preadipocyte proliferation.

\section{Influence of adiponectin on 3T3-L1 preadipocyte migration}

No cell migration was observed 6 and $12 \mathrm{~h}$ after scratching in the 10\% DMEM group, and signs of apoptosis were evident (Figure 4, panels A6 and A12), however, some migration had occurred after $24 \mathrm{~h}$ (Figure 4, panel A24). Movement of cells was seen $6 \mathrm{~h}$ after scratching in the $10 \%$ DMEM $+10 \%$ FBS group (Figure 4, panel B6) and this peaked at $12 \mathrm{~h}$ (Figure 4, panel B12). By $24 \mathrm{~h}$, cells displayed apoptosis and migration had decreased (Figure 4, panel B24). An increasing level of cell migration was observed at each time point in the $10 \%$ DMEM $+10 \% \mathrm{FBS}+25 \mu \mathrm{g} / \mathrm{mL}$ adiponectin group (Figure 4, panels C6 and C12) and the wound had 
completely healed after $24 \mathrm{~h}$ (Figure 4, panel C24). This result suggests that adiponectin can promote cell migration in 3T3-L1 preadipocytes.
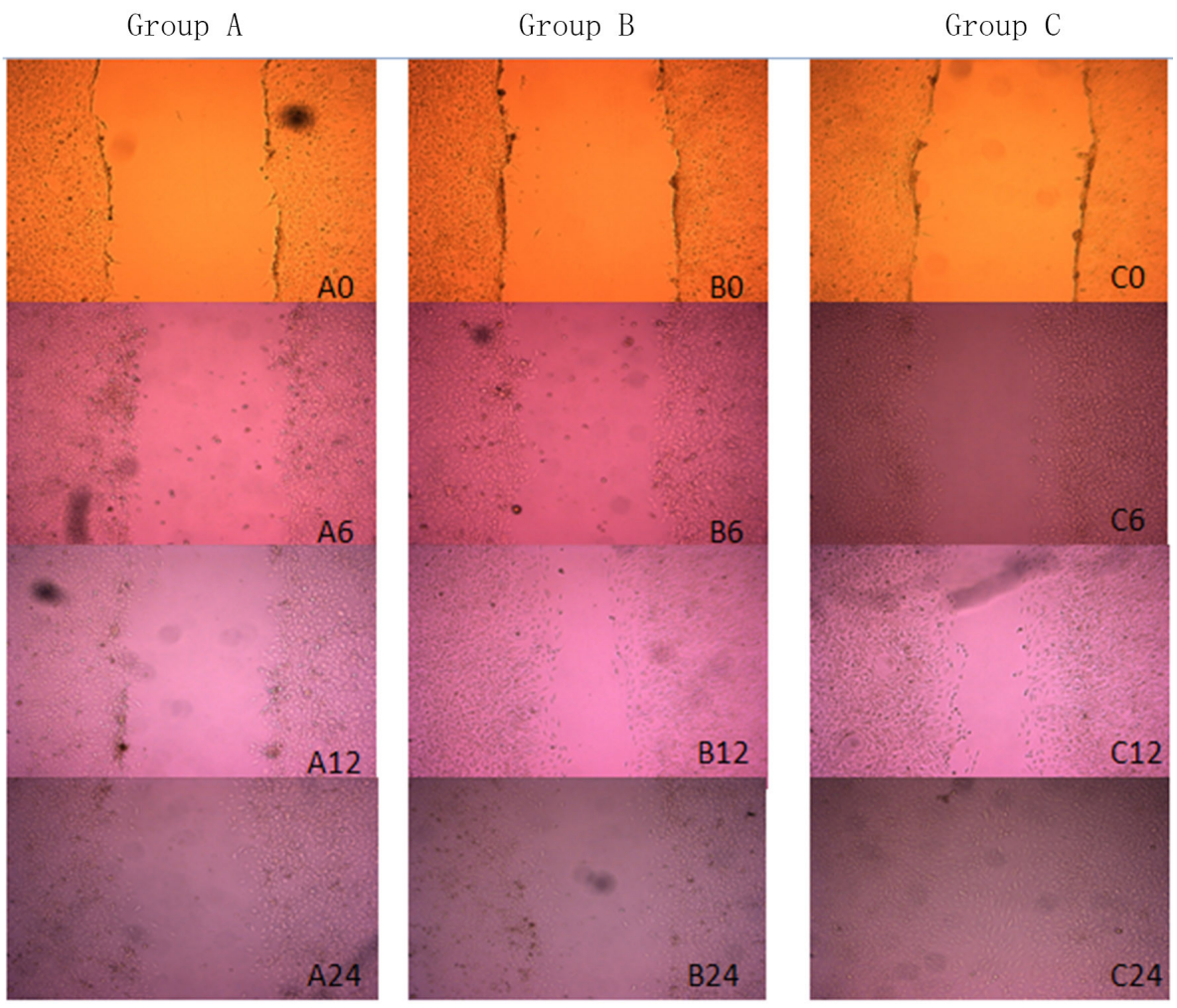

Figure 4. Influence of adiponectin on 3T3-L1 preadipocyte migration. Numbers represent $0,6,12$, and $24 \mathrm{~h}$ after being lightly scratched with an aseptic pipette tip. Group A: cells cultured in 10\% Dulbecco's modified Eagle medium (DMEM); Group B: cells cultured in 10\% DMEM $+10 \%$ fetal bovine serum (FBS); Group C: cells cultured in $10 \%$ DMEM $+10 \%$ FBS $+25 \mu \mathrm{g} / \mathrm{mL}$ adiponectin. In Group C, migration occurred continuously throughout the experiment (C0, C6, C12), with the wound appearing completely healed after $24 \mathrm{~h}(\mathrm{C} 24)$.

\section{DISCUSSION}

Preadipocytes are the precursors of adipocytes, the continuous proliferation and differentiation of which are required for ATWH. Fat necrosis is a common problem in overweight and obese patients, for whom serum adiponectin levels tend to be lower than those of healthy people (Kadowaki and Yamauchi, 2005). Adiponectin is a plasma protein secreted exclusively by adipocytes (Arita et al., 1999; Kawada and Hasegawa, 2012) and is found at physiological concentrations between 3 and $30 \mu \mathrm{g} / \mathrm{mL}$. Recently, studies on adiponectin have concentrated on insulin resistance, metabolic syndrome, and atherosclerosis (Maeda et al., 2002; Okamoto et al., 2002, 2006), while many others have established its role in wound healing in various tissues. For example, adiponectin was used to treat damage resulting from alcoholic liver disease (Ouchi et al., 2011) and ethanol-induced gastric mucosal injury (Xu et al., 2003). In addition, 
adiponectin-mediated repair of periodontal damage (Yamamoto et al., 2012) and hearing loss has been demonstrated, and it plays important roles in prostate cancer (Salathia et al., 2013) and colon carcinoma (Lu et al., 2012).

In this study, we established that AdipoR1/2 are expressed in 3T3-L1 preadipocytes using immunohistochemistry and RT-PCR. Furthermore, we showed that adiponectin concentration is not significantly affected by the density of preadipocytes, and demonstrated its ability to promote cell proliferation and migration, as well as wound healing in vitro. Therefore, we concluded that adiponectin should be conducive to the postoperative ATWH process to a certain extent.

The proliferation and migration of certain cell types depends on the targeting action of adiponectin. Globular adiponectin induces the migration of muscle satellite cells and promotes differentiation of skeletal muscle cells (Ogunwobi and Beales 2006), in addition to promoting the proliferation and differentiation of osteoblasts (Fiaschi et al., 2012). However, adiponectin appears to inhibit the growth and migration of human aortic smooth muscle cells (Luo et al., 2005) and hepatic stellate cells (Arita et al., 2002). Moreover, it modulates the expression of transforming growth factor $\beta$ and inhibits the proliferation and migration of keratinocytes, representing a potential treatment for poor wound healing in diabetic patients. However, a recent paper described the somewhat contradictory finding that adiponectin promotes the growth and migration of keratinocytes via the ERK signal pathway during wound healing in skin (Shibata et al., 2012), a conclusion consistent with our results. Yet the mechanism behind its effect on ATWH is not clear and further studies are needed. Our results suggest an optimum adiponectin concentration of $1.25 \mathrm{ng} / \mathrm{mL}$ for the promotion of preadipocyte proliferation, while its concentration in healthy people ranges from 3 to $30 \mu \mathrm{g} / \mathrm{mL}$ (Kawai et al., 2008). Therefore, physiological concentrations of adiponectin should be sufficient to promote ATWH, although this remains to be proved.

ATWH is a complex process, in which not only cell proliferation and migration, but also apoptosis and differentiation play a part (Collawn et al., 2012). Interestingly, we observed a more significant degree of apoptosis in the control groups than in the experimental group. It is unclear whether adiponectin inhibits apoptosis in 3T3-L1 preadipocytes, but the significant differences between groups suggest that it may play a role in this process, although further investigation is required. Besides, the present study consisted of only in vitro experiments. An animal model will be employed in our future study on adiponectin's function in promoting ATWH.

In conclusion, the results of this study revealed that 3T3-L1 preadipocytes express AdipoR $1 / 2$ and secrete adiponectin, which promotes their proliferation and migration. Locally or systemically administered recombinant adiponectin may improve postoperative ATWH, but further primary research and clinical studies are needed.

\section{Conflicts of interest}

The authors declare no conflict of interest.

\section{ACKNOWLEDGMENTS}

Shenzhen Special Fund for the Development of Strategic Emerging Industry (project \#JCYJ20120614085530935); the Shenzhen Knowledge Innovation Program (project funds \#JCYJ20130401114114947, \#JCYJ20130401114225775); the Project of Science and 
Technology of Shenzhen (Technological Innovation Project: \#CXZZ20120614154434310); and the Plan of the Construction of Innovative Environment in Shenzhen (Key Laboratory: \#ZDSY20130531165409949).

\section{REFERENCES}

Arita Y, Kihara S, Ouchi N, Takahashi M, et al. (1999). Paradoxical decrease of an adipose-specific protein, adiponectin, in obesity. Biochem. Biophys. Res. Commun. 257: 79-83.

Arita Y, Kihara S, Ouchi N, Maeda K, et al. (2002). Adipocyte-derived plasma protein adiponectin acts as a plateletderived growth factor-BB-binding protein and regulates growth factor-induced common postreceptor signal in vascular smooth muscle cell. Circulation 105: 2893-2898.

Cheng B, Liu HW and Fu XB (2011). Update on pruritic mechanisms of hypertrophic scars in postburn patients: the potential role of opioids and their receptors. J. Burn Care Res. 32: e118-125.

Collawn SS, Banerjee NS, de la Torre J, Vasconez L, et al. (2012). Adipose-derived stromal cells accelerate wound healing in an organotypic raft culture model. Ann. Plast. Surg. 68: 501-504.

Fiaschi T, Giannoni E, Taddei ML and Chiarugi P (2012). Globular adiponectin activates motility and regenerative traits of muscle satellite cells. PLoS One 7: e34782.

Jeon IH, Morrey BF, Anakwenze OA and Tran NV (2011). Incidence and implications of early postoperative wound complications after total elbow arthroplasty. J. Shoulder Elbow Surg. 20: 857-865.

Kadowaki T and Yamauchi T (2005). Adiponectin and adiponectin receptors. Endocr. Rev. 26: 439-451.

Kamada Y, Tamura S, Kiso S, Matsumoto H, et al. (2003). Enhanced carbon tetrachloride-induced liver fibrosis in mice lacking adiponectin. Gastroenterology 125: 1796-1807.

Kawada T and Hasegawa M (2012). Predictive ability of serum high-molecular-weight adiponectin in combination with serum insulin and serum C-reactive protein for the presence of metabolic syndrome. Ann. Hum. Biol. 39: 108-112.

Kawai K, Kageyama A, Tsumano T, Nishimoto S, et al. (2008). Effects of adiponectin on growth and differentiation of human keratinocytes - implication of impaired wound healing in diabetes. Biochem. Biophys. Res. Commun. 374: 269-273.

Liang GP, Su YY, Chen J, Yang ZC, et al. (2009). Analysis of the early adaptive response of endothelial cells to hypoxia via a long serial analysis of gene expression. Biochem. Biophys. Res. Commun. 384: 415-419.

Lu F, Li J, Gao J, Ogawa R, et al. (2009). Improvement of the survival of human autologous fat transplantation by using VEGF-transfected adipose-derived stem cells. Plast. Reconstr. Surg. 124: 1437-1446.

Lu JP, Hou ZF, Duivenvoorden WC, Whelan K, et al. (2012). Adiponectin inhibits oxidative stress in human prostate carcinoma cells. Prostate Cancer Prostatic Dis. 15: 28-35.

Luo XH, Guo LJ, Yuan LQ, Xie H, et al. (2005). Adiponectin stimulates human osteoblasts proliferation and differentiation via the MAPK signaling pathway. Exp. Cell Res. 309: 99-109.

Maeda N, Shimomura I, Kishida K, Nishizawa H, et al. (2002). Diet-induced insulin resistance in mice lacking adiponectin/ ACRP30. Nat. Med. 8: 731-737.

Ogunwobi OO and Beales IL (2006). Adiponectin stimulates proliferation and cytokine secretion in colonic epithelial cells. Regul. Pept. 134:105-113.

Okamoto Y, Kihara S, Ouchi N, Nishida M, et al. (2002). Adiponectin reduces atherosclerosis in apolipoprotein E-deficient mice. Circulation 106: 2767-2770.

Okamoto Y, Kihara S, Funahashi T, Matsuzawa Y, et al. (2006). Adiponectin: a key adipocytokine in metabolic syndrome. Clin. Sci. 110: 267-278.

Ouchi N, Parker JL, Lugus JJ and Walsh K (2011). Adipokines in inflammation and metabolic disease. Nat. Rev. Immunol. 11: 85-97.

Pradhan L, Cai X, Wu S, Andersen ND, et al. (2011). Gene expression of pro-inflammatory cytokines and neuropeptides in diabetic wound healing. J. Surg. Res. 167: 336-342.

Salathia NS, Shi J, Zhang J and Glynne RJ (2013). An in vivo screen of secreted proteins identifies adiponectin as a regulator of murine cutaneous wound healing. J. Invest. Dermatol. 133: 812-821.

Shibata S, Tada Y, Asano Y, Hau CS, et al. (2012). Adiponectin regulates cutaneous wound healing by promoting keratinocyte proliferation and migration via the ERK signaling pathway. J. Immunol. 189: 3231-3241.

Xu A, Wang Y, Keshaw H, Xu LY, et al. (2003). The fat-derived hormone adiponectin alleviates alcoholic and nonalcoholic fatty liver diseases in mice. J. Clin. Invest. 112: 91-100.

Yamamoto S, Watabe K, Araki H, Kamada Y, et al. (2012). Protective role of adiponectin against ethanol-induced gastric injury in mice. Am. J. Physiol. Gastrointest. Liver Physiol. 302: G773-G780. 